\title{
Effectiveness of a comprehensive post-operative health education program in improving quality of life after gastric cancer surgery
}

\author{
Qian Gao ${ }^{1 \#}$, Huiyan $\mathrm{Li}^{2 \#}$, Yuan $\mathrm{Zou}^{1}$, Bo Hou ${ }^{1}$, Lixiu Liu ${ }^{3}$ \\ ${ }^{1}$ Second Ward, Department of Gastrointestinal Surgery, ${ }^{2}$ Department of Nursing, ${ }^{3}$ Second Ward, Department of Colorectal Surgery, Harbin Medical \\ University Cancer Hospital, Harbin 150081, China \\ Contributions: (I) Conception and design: Q Gao, L Liu; (II) Administrative support: Q Gao, H Li, B Hou; (III) Provision of study materials: H Li, Y \\ Zou, B Hou; (IV) Collection and assembly of data: Q Gao, H Li, L Liu; (V) Data analysis and interpretation: Q Gao, Y Zou, B Hou; (VI) Manuscript \\ writing: All authors; (VII) Final approval of manuscript: All authors. \\ \#These authors contributed equally to this work. \\ Correspondence to: Lixiu Liu. Department of Colorectal Surgery, Harbin Medical University Tumor Hospital, Nangang District, Harbin City, Harbin \\ 150081, China. Email: lihuiyanlhyy@sina.com.
}

Background: Patients with gastric cancer often experience postoperative problems such as dumping syndrome, abdominal cramps, and nausea, which have profound effects on their mental health and quality of life. Earlier studies have shown that comprehensive health education can dramatically improve the quality of life of cancer patients after surgery. Thus, in this study, we conducted a health education intervention program in patients who had undergone surgical treatment for gastric cancer and evaluated the effectiveness of this program in improving the postoperative quality of life, with an attempt to offer evidence for better postoperative management of gastric cancer patients.

Methods: Totally, 80 patients with gastric cancer who underwent surgery at our center from February 2018 to February 2019 were equally divided into an observation group and control group according to the random number table method. The observation group was offered with interventions regarding health education, including disease awareness-raising, instructions on behavior and lifestyle, rehabilitation management, and mental health counseling, and the control group received the routine nursing intervention. The quality of life one month before and after surgery was assessed by using the Chinese version of the European Organization for Research and Treatment of Cancer (EORTC) QLQ-STO22.

Results: The baseline data and quality of life showed no significant differences between the observation group and the control group one month before surgery (both $\mathrm{P}>0.05$ ). One month after the surgery, the scores of dysphagia, pain/discomfort, reflux symptoms, and emotional issues and overall score in the observation group decreased significantly $(34.86 \pm 2.170,38.66 \pm 3.08,32.26 \pm 2.76,49.55 \pm 4.20$, and 54.26 \pm 7.03 , respectively), which were also significantly lower than those in the control group $(36.33 \pm 3.10,44.29 \pm 3.72$, $33.64 \pm 3.10,53.56 \pm 3.25$, and $60.17 \pm 7.28$, respectively; all $\mathrm{P}<0.05)$.

Conclusions: The comprehensive health education intervention program can effectively improve the quality of life in patients after gastric cancer surgery and deserves further application in clinical settings.

Keywords: Gastric cancer; health education; intervention; factors

Submitted Jan 17, 2020. Accepted for publication Mar 25, 2020.

doi: $10.21037 /$ apm.2020.04.14

View this article at: http://dx.doi.org/10.21037/apm.2020.04.14 


\section{Introduction}

Gastric cancer is a common malignant gastrointestinal condition. China is among the countries with the highest incidence of gastric cancer. The prevalence rates of gastric cancer vary dramatically between different geographical regions; in general, gastric cancer is seen much more frequently in China's northwest and eastern coastal areas than in Southern China (1). At present, surgery is the mainstay of treatment for gastric cancer, although radiotherapy and chemotherapy can also be helpful (2). Although surgery can effectively relieve symptoms, patients often experience symptoms such as dumping syndrome, abdominal cramps, nausea, diarrhea, anemia, vitamin deficiency, and weight loss after surgery (3). Meanwhile, patients can develop a series of psychological symptoms due to the diagnosis of cancer itself, the postoperative complications, and the side effects of radiotherapy and chemotherapy (4). As a result, the quality of life after surgery is often severely impaired due to these physiological and psychological changes (5). Previous studies have found that comprehensive health education provided to cancer patients after surgery can markedly improve patients' quality because it has many advantages: it can increase the patients' awareness of disease and late complications; guidance on lifestyle and diets can increase the patients' selfcare skills, and health education on the families can create supportive familial and social environments. Thus, in this study, we conducted a health education intervention program in patients who had undergone surgical treatment for gastric cancer and evaluated the effectiveness of this program in improving the postoperative quality of life, with an attempt to offer evidence for better postoperative management of gastric cancer patients.

\section{Methods}

\section{Subjects}

In this study were enrolled a total of 80 patients with gastric cancer that underwent surgery from February 2018 to February 2018 at our center. The inclusion criteria were as follows: (I) aged 35-75 years; (II) meeting the diagnostic criteria for gastric cancer (6); (III) with regular communication and comprehension abilities; (IV) be aware of their disease. Exclusion criteria: (I) with other chronic diseases such as cardiovascular disease; and (II) with mental illness or disturbance of consciousness. The Ethics Committee approved the study of our center. All patients signed informed consent. A total of 80 patients were included, and they were equally divided into the observation group ( $n=40)$ and the control group $(n=40)$ according to the random number table method.

\section{Interventions}

Patients in the control group received routine nursing interventions, including preoperative gut preparation, intravenous administration of antibiotics, and monitoring of postoperative complications such as pneumonia, infection, bleeding, and anal fistula.

A comprehensive health education program was offered to patients in the observation group. The program was carried out by two uniformly trained nurses, with the primary interventions including (I) Disease awareness-raising activities: after gastrectomy was performed and the disease condition became stable, face-to-face health education was offered to the patient with the presence of his/her family member(s), during which the etiology of the disease, possible complications after the surgery, critical points of rehabilitation management, and further treatment options were explained to the patient. (II) Guidance on behavior and lifestyle: to distribute self-developed health education manuals and pamphlets were distributed to the patients, delivering knowledge on sleeping, exercise, diets, and nutritional needs; patients have also been instructed how to use those materials. (III) Rehabilitation management: Rehabilitation experts and clinicians were invited to communicate with the patients about post-operative self-care, taking medications according to doctor's orders, and the importance of regular followup visits. (IV) Psychological counseling: The curability of disease and the importance of family support were explained via face-to-face communication with patients and their family members. Based on the health education during the hospital stay, a follow-up plan was established, during which three telephone-based follow-up visits were conducted two weeks after discharge to ensure that patients followed the instructions.

\section{Evaluating the effectiveness of the program}

\section{Questionnaires}

The quality of life one month before and after surgery was assessed by using the Chinese version of the European Organisation for Research and Treatment of Cancer (EORTC) QLQ-STO22 (7). Eight problems, including difficulty swallowing, pain, discomfort, eating restrictions, reflux symptoms, emotional issues, dry mouth, body image, 
Table 1 Sociodemographic and disease characteristics in two groups [n (\%)]

\begin{tabular}{|c|c|c|c|c|}
\hline Characteristics & Observation group & Control group & $\chi^{2}$ & $\mathrm{P}$ \\
\hline Males & $33(82.50)$ & $29(72.50)$ & & \\
\hline Females & $7(17.50)$ & $11(27.50)$ & & \\
\hline Age, years & & & 2.650 & 0.104 \\
\hline$\geq 65$ & $11(27.50)$ & $18(45.00)$ & & \\
\hline Marital status & & & 2.051 & 0.152 \\
\hline Married & $30(75.00)$ & $35(87.50)$ & & \\
\hline Others (unmarried/divorced/widowed) & $10(25.00)$ & $5(12.50)$ & & \\
\hline High school or above & $35(87.50)$ & $32(80.00)$ & & \\
\hline Gastric cancer staging & & & 2.650 & 0.104 \\
\hline I-II & $18(45.00)$ & $11(27.50)$ & & \\
\hline III-IV & $22(55.00)$ & $29(72.50)$ & & \\
\hline Gastric cancer typing & & & 0.558 & 0.757 \\
\hline Diffuse type & $24(60.00)$ & $22(55.00)$ & & \\
\hline Intestinal type & $11(27.50)$ & $14(35.00)$ & & \\
\hline Mixed type & $5(12.50)$ & $4(10.00)$ & & \\
\hline
\end{tabular}

and hair loss were scored on a 4-point scale (1 to 4 points for asymptomatic to very severe symptoms, respectively). High scores are severe problems, showing an inferior quality of life.

\section{Evaluation of the effectiveness}

The baseline survey was performed after the patient's condition became stable and before discharge. The survey was conducted face-to-face by two uniformly trained nurses. The effectiveness evaluation was performed one month after discharge; for this purpose, the patient was invited to the hospital to receive routine review examinations and a second survey. A small number of patients who did not come to the hospital were surveyed via telephone.

\section{Statistical analysis}

Statistical analysis was performed using SPSS 19.0 software package. Count data are presented with a rate (\%) and analyzed by the chi-square test. The QLQ-STO22 scores are presented as $\bar{x} \pm s$; the pre- and post-operative scores were compared by using paired $t$-test, and the inter-group comparisons were based on independent samples $t$-test. A P value of $<0.05$ was considered significantly different.

\section{Results}

\section{Sociodemographic and disease characteristics}

Among these 80 patients, there were 62 males and 18 females aged $42-75$ years (mean: $60.28 \pm 6.10$ years). The sociodemographic and disease characteristics showed no significant difference between these two groups (all $\mathrm{P}>0.05$ ) (Table 1).

\section{QLQ-STO22 scores before and after surgery}

The scores of dysphagia, pain/discomfort, reflux symptoms, 
Table 2 QLQ-STO22 scores before and after surgery in two groups $(\bar{x} \pm s$, points)

\begin{tabular}{|c|c|c|c|c|c|c|c|c|}
\hline Problems & \multicolumn{4}{|c|}{ One month before surgery } & \multicolumn{4}{|c|}{ One month after surgery } \\
\hline Dysphagia & $40.24 \pm 4.46$ & $38.80 \pm 4.52$ & 1.434 & 0.156 & $34.86 \pm 2.17^{\star}$ & $36.33 \pm 3.10^{*}$ & 2.457 & 0.016 \\
\hline Pain/discomfort & $46.30 \pm 5.78$ & $48.54 \pm 5.64$ & 1.754 & 0.083 & $38.66 \pm 3.08^{\star}$ & $44.29 \pm 3.72^{\star}$ & 7.373 & $<0.001$ \\
\hline Eating restrictions & $34.09 \pm 2.92$ & $34.48 \pm 3.07$ & 0.582 & 0.562 & $33.16 \pm 2.06$ & $33.52 \pm 2.26$ & 0.745 & 0.459 \\
\hline Emotional issues & $56.85 \pm 4.66$ & $58.20 \pm 4.78$ & 1.279 & 0.205 & $49.55 \pm 4.20^{*}$ & $53.56 \pm 3.25^{\star}$ & 4.776 & $<0.001$ \\
\hline Dry mouth & $33.33 \pm 4.85$ & $34.10 \pm 4.78$ & 0.715 & 0.477 & $31.42 \pm 4.28$ & $32.86 \pm 3.10$ & 1.723 & 0.089 \\
\hline Body image & $28.29 \pm 3.86$ & $29.23 \pm 3.98$ & 1.072 & 0.287 & $26.86 \pm 3.56$ & $27.85 \pm 3.41$ & 1.270 & 0.208 \\
\hline Hair loss & $32.18 \pm 4.07$ & $32.25 \pm 4.02$ & 0.077 & 0.939 & $30.58 \pm 3.48$ & $31.55 \pm 4.00$ & 1.157 & 0.251 \\
\hline
\end{tabular}

*, $\mathrm{P}<0.05$, compared with the pre-operative score.

and emotional issues and the total score significantly decreased after surgery in both groups (all $\mathrm{P}<0.05$ ); besides, the scores of dysphagia, pain/discomfort, reflux symptoms, and emotional issues and the total score were significantly lower in the observation group than in the control group (all $\mathrm{P}<0.05)$ (Table 2).

\section{Discussion}

Gastric cancer is a common malignancy in China. According to data released by The National Central Cancer Registry (NCCR) of China (8), approximately 424,000 new gastric cancer cases and 298,000 cancer deaths occurred in China in 2012, accounting for nearly half of the global new cases and deaths. The postoperative survival rate of patients with gastric cancer has dramatically increased with the advances in treatments for gastric cancer; however, the post-operative complications have severely impaired the quality of life of these patients (9). As a critical part of postoperative management, ongoing health education helps to promote the physical and mental rehabilitation of surgical patients and improve the quality of life.

Just like many patients with other cancers, patients with gastric cancer often require multi-faceted health education needs due to the psychological stress and physical changes caused by having cancer itself and after experiencing a variety of treatment-related complications (10). Health education should be done to tailor comprehensive health education to the psychological and mental status, diet and lifestyle. Meanwhile, the content of education should also be individualized based on the location of cancer. Therefore, one single intervention method often can not effectively to improve the quality of life of a specific patient. Lee et al. (11) carried out a Tai Chi-based self-care education program in patients with gastric cancer two years after surgery, and the results showed that although the patients generally felt better after the intervention, the quality of life was not significantly improved, highlighting the importance of a more comprehensive health education program. In our current study, we firstly increased the patients' awareness of the disease and the treatment-related complications, followed by the enhanced guidance of behavior and lifestyle and the counseling of psychological and emotional issues. Expert lectures were arranged to emphasize the importance of medication adherence and regular follow-up. Finally, the patients and their families were informed about the role of family support during rehabilitation. Evaluation of the effectiveness of this comprehensive health education program showed that, compared with the control group, the observation group had significantly lower scores of dysphagia, pain/discomfort, reflux symptoms, and emotional issues and significantly lower total scores one month after surgery, indicating that the patients' quality of life was remarkably improved.

Also, most earlier health education programs were conducted inside hospitals, and there was a lack of followup data after discharge. Although evaluations in these studies typically revealed an improvement in the quality of life of the surgical patients, it might be explained by the following fact: during the hospital stay, the patients usually 
can receive and feel high levels of family, and social supports and they can receive timely guidance and encouragement from medical staff; as a result, they often report high quality of life. However, as shown in some studies (12), the family support gradually decreases after the patients are discharged, and some postoperative complications occur, leading to the decline of the quality of life. Therefore, strengthening the follow-up visits and offering continuous guidance after discharge will help to improve the self-care ability of patients after discharge, helping them to develop reasonable behaviors and lifestyle and increase treatment compliance, which is particularly valuable for improving their quality of life. In our current study, we offered continuous health education for patients after being discharged via telephone follow-up. The quality of life of surgical patients remained at an elevated level one month after discharge. Therefore, the combination of in-hospital face-to-face health education and follow-up health education after discharge is an effective strategy for improving the quality of life that can be readily accepted by patients.

In summary, the comprehensive health education program is an effective method to improve the quality of life of patients with gastric cancer surgery and calls for further application in clinical settings.

\section{Acknowledgments}

Funding: Key Project of Haiyan Project of Harbin Medical University Cancer Hospital (JJZD2018-07).

\section{Footnote}

Conflicts of Interest: All authors have completed the ICMJE uniform disclosure form (available at http://dx.doi. org/10.21037/apm.2020.04.14). The authors have no conflicts of interest to declare.

Ethical Statement: The authors are accountable for all aspects of the work in ensuring that questions related to the accuracy or integrity of any part of the work are appropriately investigated and resolved. The Ethics Committee approved the study of our center (No. KY201814). All patients signed informed consent.

Open Access Statement: This is an Open Access article distributed in accordance with the Creative Commons Attribution-NonCommercial-NoDerivs 4.0 International License (CC BY-NC-ND 4.0), which permits the non- commercial replication and distribution of the article with the strict proviso that no changes or edits are made and the original work is properly cited (including links to both the formal publication through the relevant DOI and the license). See: https://creativecommons.org/licenses/by-nc-nd/4.0/.

\section{References}

1. Tan J, Yang B, Xu Z, et al. Tumor deposit indicates worse prognosis than metastatic lymph node in gastric cancer: a propensity score matching study. Ann Transl Med 2019;7:671.

2. Guner A, Hyung WJ. Advantages of Splenic Hilar Lymph Node Dissection in Proximal Gastric Cancer Surgery. J Gastric Cancer 2020;20:19-28.

3. Stewart C, Chao J, Chen YJ, et al. Multimodality management of locally advanced gastric cancer-the timing and extent of surgery. Transl Gastroenterol Hepatol 2019;4:42.

4. Shinde A, Novak J, Amini A, et al. The evolving role of radiation therapy for resectable and unresectable gastric cancer. Transl Gastroenterol Hepatol 2019;4:64.

5. Yang FY, Guo YJ, Jin Y, et al. Investigation of postoperative life quality and mental health of patients with gastric cancer and the effects of interventions. Progress in Modern Biomedicine 2014;14:4150-2.

6. National "863" Major Project "Molecular Typing and Individualized Diagnosis and Treatment of Gastric Cancer" Working Group. Recommendations on pathological typing and diagnostic criteria of gastric cancer. Chinese Journal of Pathology 2010;39:266-9.

7. Meng Q, Luo JH, Wan CH, et al. Development and evaluation on Chinese version of measurement instrument of quality of life for patients with stomach cancer: EORTC QLQ-STO22. Journal of Kunming Medical University 2012;33:34-8.

8. Zuo TT, Zheng RS, Zeng HM, et al. Epidemiology of stomach cancer in China. Chinese Journal of Clinical Oncology 2017;44:52-8.

9. Jin Z, Ma L, Xiaokaiti A, et al. Correlation of complication with survival and quality of life in elderly gastric patients after postoperative surgery. Chinese Journal of Clinical Oncology and Rehabilitation 2015;22:145-8.

10. Zhou QL. Effect evaluation of homogeneous health education model on nursing care among postoperative patients with gastric cancer. Chinese Journal of Health Education 2016;32:281-4.

11. Lee EO, Chae YR, Song R, et al. Feasibility and effects of 
a tai chi self-help education program for Korean gastric cancer survivors. Oncology Nursing Forum 2010;37:E1-6.

12. Ren YN, Yu ZM, Dong SB. Effects of continuing nursing health education on postoperative rehabilitation of patients with gastric cancer. Chinese Journal of Modern Nursing 2016;22:766-9.

Cite this article as: Gao Q, Li H, Zou Y, Hou B, Liu L. Effectiveness of a comprehensive post-operative health education program in improving quality of life after gastric cancer surgery. Ann Palliat Med 2020;9(3):921-926. doi: 10.21037/ apm.2020.04.14 